\title{
Zolpidem reduces pain intensity postoperatively: a systematic review and meta-analysis of the effect of hypnotic medicines on post-operative pain intensity
}

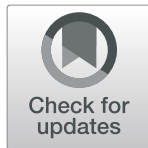

Edel T. O'Hagan ${ }^{1,2^{*}}$ (D) Markus Hübscher ${ }^{1,2}$, Christopher B. Miller ${ }^{3}$, Christopher J. Gordon ${ }^{3,4}$, Sylvia Gustin ${ }^{1,5}$, Nancy Briggs ${ }^{6}$ and James H. McAuley ${ }^{1,7}$

\begin{abstract}
Background: This systematic review aimed to investigate whether the administration of hypnotic medicines, zdrugs, melatonin or benzodiazepines, reduced pain intensity postoperatively.

Methods: Medline, Embase, Cinahl, Psych info, Central and PubMed databases were searched, from inception to February 2020 to identify relevant trials. The search was extended, post hoc, to include meta-Register of Controlled Trials, the Web of Science and the conference booklets for the 14th, 15th, and 16th International Association for the Study of Pain conferences. Two independent reviewers screened titles and abstracts and cross-checked the extracted data.

Results: The search retrieved 5546 articles. After full-text screening, 15 trials were included, which had randomised 1252 participants. There is moderate-quality evidence that in the short-term [WMD $-1.06, \mathrm{Cl}-1.48$ to $-0.64, p \leq$ $.01]$ and low-quality evidence that in the medium-term [WMD $-0.90, \mathrm{Cl}-1.43$ to $-0.37, p \leq .01]$ postoperative period oral zolpidem $5 / 10 \mathrm{mg}$ with other analgesic medicines reduced pain intensity compared to the same analgesic medicines alone.

There is low-quality evidence that melatonin was not effective on postoperative pain intensity compared to placebo. The results of benzodiazepines on pain intensity were mixed. The authors reported no significant adverse events.

Conclusions: There is promising evidence that the hypnotic medicine zolpidem, adjuvant to other analgesics, is effective at achieving a minimally clinically important difference in pain intensity postoperatively. There is no consistent effect of melatonin or benzodiazepines on postoperative pain intensity. Readers should interpret these results with some caution due to the lack of data on safety, the small number of trials included in the pooled effects and their sample sizes.
\end{abstract}

Systematic review registration: The protocol for this systematic review was registered with PROSPERO ID= CRD42015025327.

\footnotetext{
* Correspondence: e.ohagan@neura.edu.au

${ }^{1}$ Centre for Pain IMPACT, Neuroscience Research Australia, Randwick, NSW

2031, Australia

${ }^{2}$ Prince of Wales Clinical School, The University of New South Wales,

Randwick, NSW 2031, Australia

Full list of author information is available at the end of the article
}

(c) The Author(s). 2020 Open Access This article is licensed under a Creative Commons Attribution 4.0 International License, which permits use, sharing, adaptation, distribution and reproduction in any medium or format, as long as you give appropriate credit to the original author(s) and the source, provide a link to the Creative Commons licence, and indicate if changes were made. The images or other third party material in this article are included in the article's Creative Commons licence, unless indicated otherwise in a credit line to the material. If material is not included in the article's Creative Commons licence and your intended use is not permitted by statutory regulation or exceeds the permitted use, you will need to obtain permission directly from the copyright holder. To view a copy of this licence, visit http://creativecommons.org/licenses/by/4.0/ The Creative Commons Public Domain Dedication waiver (http://creativecommons.org/publicdomain/zero/1.0/) applies to the data made available in this article, unless otherwise stated in a credit line to the data. 


\section{Introduction}

Postoperative pain is common; out of the estimated 48 million surgical procedures performed each year in the USA, $80 \%$ of patients report significant or severe pain in the postoperative period [1]. Acute postoperative pain is associated with decreased patient mobility, which can lead to an increased risk of complications such as deep vein thrombosis, pulmonary embolus and pneumonia [2]. Postoperative pain can also result in an extended hospital stay [2,3], increase the risk of readmission $[2,3]$ and delay return to normal function and work [3]. Up to $50 \%$ of patients develop persistent postoperative pain [4], which is debilitating and can have legal and medico-economic consequences [5]. A consistent and strong predictor of persistent postoperative pain is intensity in the immediate postoperative period [6].

The evidence for the effectiveness of analgesic interventions for postoperative pain is typically low quality [7]. Only 4 out of 32 recommendations in the American Pain Society guideline for managing acute postoperative pain were supported by high-quality evidence [7]. Multimodal analgesia that includes opioids is recommended [7]. There is evidence that postoperative administration of opioids increases the risk of long-term opioid use following common surgical procedures such as total knee replacement (TKR) and laparoscopic cholecystectomy [8]. A recent Lancet series [9-11] highlighted the important role of non-opioid and opioid-sparing pharmacological interventions for the management of postoperative pain.

Following major operations, patients commonly report poor sleep quality [11]. Almost half (42\%) of patients report unsatisfactory sleep after orthopaedic, vascular and general surgery [12]. Poor sleep quality is commonly managed with hypnotic medicines, including $\mathrm{z}$-drugs, melatonin and benzodiazepines. These medicines may be provided postoperatively to improve sleep quality.

There is evidence that sleep quality and pain intensity have a bi-directional relationship [13]. For example, sleep quality was found to be associated with next day pain intensity, and daytime pain intensity was found to be associated with that night's sleep quality for people with low back pain. These effects were independent of pain duration, depression and anxiety [13]. Given this relationship, it is possible that hypnotic medicines administered postoperatively to improve sleep quality may lead to reduced pain intensity and persistent postoperative pain. This effect has never been systematically evaluated. To our knowledge, this is the first systematic review to investigate the effect of hypnotic medicines on postoperative pain intensity.

The primary aim of this systematic review was to determine whether hypnotic medicines reduce postoperative pain intensity.

The key objectives were to determine whether hypnotic medicines:
- Reduce postoperative pain intensity,

- Reduce opioid consumption,

- Improve postoperative sleep outcomes.

We were also interested in whether any effects of hypnotic medicines on postoperative pain intensity are moderated by:

- The duration of symptoms,

- The type of surgery.

\section{Methods}

\section{Protocol and registration}

The study is reported in accordance with the PRISMA statement (Additional file 1) for the reporting of systematic reviews and meta-analyses [14], and the protocol was registered with PROSPERO ID=CRD42015025327 http://www.crd.york.ac.uk/PROSPERO/display_record. asp?ID=CRD42015025327.

\section{Eligibility criteria}

Published randomised and quasi-randomised controlled trials (RCTs) from database inception, in any language, were considered for inclusion in the review. Inclusion criteria were defined using the PICO (Patients, Intervention, Control, Outcome) framework [14].

\section{Patients}

Trials that included adults older than 18 years of age who had undergone any surgery were eligible for the review. Trials that included a mixed sample of postoperative pain and other pain, such as low back pain, were excluded unless results for the postoperative sample were reported or could be obtained, separately.

\section{Intervention}

Trials that tested the effects of z-drugs ((e.g. zolpidem and zopiclone) a group of non-benzodiazepine hypnotics) [15], melatonin or benzodiazepine medicines, administered postoperatively as monotherapy or with analgesic interventions were eligible for inclusion. Trials were eligible if the hypnotic medicine was administered by oral, intravenous (IV), intramuscular (IM) or intranasal routes. Trials with an epidural or neuraxial mode of administration were excluded due to possible safety concerns. The most recent guideline for the management of postoperative pain states that the "neuraxial administration of benzodiazepines...in the treatment of postoperative pain is not recommended because of no clear benefit and insufficient evidence to determine safety" [7].

\section{Comparison}

Trials that compared a hypnotic medicine to (1) placebo, (2) analgesics (e.g. paracetamol, non-steroidal 
anti-inflammatory drugs (NSAIDs), opioids), (3) nonpharmacological modalities (e.g. cognitive-behavioral therapy-Insomnia (CBT-I), acupuncture, etc.) were included.

\section{Outcomes}

The primary outcome was pain intensity measured at any timepoint postoperatively. Trials that measured pain intensity using a valid and reliable assessment such as numeric rating scales (NRS) or visual analogue scales (VAS) were included.

Related outcomes included additional analgesia or narcotic consumption, measured as an exact dose or as the number of participants who requested additional analgesia.

Secondary outcomes are described in Additional file 2.

\section{Information sources}

Sensitive search strategies were developed for Ovid MEDLINE, Ovid Embase, Cinahl, Psych info, Central and PubMed. Databases were searched from inception to July 2015 and updated in February 2020. Post hoc, the search was extended to include meta-Register of Controlled Trials and the Web of Science and the conference booklets for the 14th, 15th and 16th International Association for the Study of Pain conferences. The search strategy was adapted from a Cochrane review on postoperative pain and a separate review on hypnotic and sedating medicines [16, 17], modified to exclude search terms for sedating medicines. The MEDLINE search strategy is provided as Additional file 3 .

\section{Study selection}

Two independent reviewers screened titles and abstracts to identify trials that met the inclusion criteria ( $\mathrm{EO}$ and either MH, CM, SG or CG). Disagreements between reviewers were resolved by discussion and consensus. Any remaining disagreements were resolved by consulting a third reviewer (JMcA). If the abstract was unclear, the article was retrieved, and two other independent reviewers reviewed the full text.

\section{Data collection process}

One review author (EO) used a standardised report form to extract data from the eligible full-text trials. The extracted data were cross-checked by two reviewers $(\mathrm{MH}$ and JMcA).

\section{Data items}

Extracted data included information on trial design and funding, recruitment source, patient characteristics, intervention, control, outcome measure assessed, duration of follow-up and results.

\section{Risk of bias in individual trials}

One reviewer (EO) applied the Cochrane tool for assessing risk of bias [18] to each trial, and the score was reviewed by a second independent reviewer $(\mathrm{MH}, \mathrm{CM}$, SG or CG). A score of 1 was allocated if there was no or a low-risk of bias for each section, 0 if there was a high risk of bias, and "unclear" if the information was not clear from the manuscript. Disagreements between reviewers were resolved by discussion. The following domains were considered: random sequence generation (selection bias), allocation concealment (selection bias), blinding of participants, blinding of personnel/care providers (performance bias), blinding of outcome assessor (detection bias), incomplete outcome data (attrition bias), selective reporting (reporting bias), group similarity at baseline (selection bias), co-interventions (performance bias), compliance (performance bias), intention-to-treatanalysis attrition bias), timing of outcome assessments (detection bias), other bias which considered industry funding and ethics approval.

\section{Summary measures}

Mean between-group differences and standard deviations for all outcomes were extracted from the manuscripts. For pain intensity, the primary outcome, if data were not reported on a $0-10$ point scale, they were converted, where possible [14], i.e. outcome measures that used $1-4$ scales were multiplied by 2.5 , and $0-100$ scales were divided by 10 [14]. Data were extracted for: immediate (up to $48 \mathrm{~h}$ postoperatively), short-term ( $48 \mathrm{~h}$ to 7 days postoperatively), medium-term (7-30 postoperatively) and long-term (greater than 1 month postoperatively) periods.

\section{Synthesis of results Main effects of hypnotic medicines}

The results from trials that were clinically homogenous were combined in a random-effects meta-analysis, and the weighted mean difference (WMD) was calculated using the RevMan review manager software, 5.3 [19]. Clinical homogeneity was determined by similarity of drug, mode of administration and control group.

\section{Sub-group analyses}

The different postoperative periods of analyses were combined into a single meta-regression analysis. We used random-effects meta-regression to investigate the relationship between pain and (1) hypnotic medicines over time, (2) control drug over time and (3) route of administration over time. A random effect for trial was included, as well as fixed effects of time and variable of interest (drug, control or route) and the interaction. For analyses involving a significant time*effect interaction, estimates of the effect size over time were obtained. 
Analyses were performed using the metafor package (version 3.4.3) in $\mathrm{R}$ [20].

We used the GRADE approach to assess the overall quality of the evidence for each outcome, as recommended in the Cochrane Handbook for Systematic Reviews of Interventions [18]. In line with this approach, we considered five factors for rating the quality of the evidence from high to no or very low-quality evidence. The five factors were (1) study design and risk of bias (downgraded if more than $25 \%$ of the participants were from studies with a high risk of bias), (2) inconsistency of results (downgraded if significant heterogeneity was present by visual inspection or if the $I^{2}$ value was greater than $50 \%$ ), (3) indirectness (generalisability of the findings; downgraded if more than $50 \%$ of the participants were outside the target group), (4) imprecision (downgraded if fewer than 400 participants were included in the comparison for continuous data and there were fewer than 300 events for dichotomous data) [21] and (5) other factors (e.g. reporting bias, publication bias).

High-quality evidence There were consistent findings among at least $75 \%$ of RCTs with low-risk of bias, consistent, direct and precise data and no known or suspected publication biases. Further research is unlikely to change either the estimate or our confidence in the results.

Moderate-quality evidence One of the domains is not met. Further research is likely to have an important impact on our confidence in the estimate of effect and may change the estimate.

Low-quality evidence Two of the domains are not met. Further research is very likely to have an important impact on our confidence in the estimate of effect and is likely to change the estimate.

Very low-quality evidence Three of the domains are not met. We are very uncertain about the results.

No evidence No RCTs were identified that addressed this outcome.

\section{Sensitivity analyses}

A sensitivity analysis was planned to determine whether excluding trials of lower methodological quality or higher risk of bias affected the effects of the group comparisons. A post hoc sensitivity analysis was conducted to assess the effect of increasing the "immediate period" to $72 \mathrm{~h}$ postoperatively.

\section{Results}

Study flow

The search identified 5546 articles. Once duplicates and trials that did not meet inclusion criteria were removed, 72 articles remained. After full-text screening, 15 trials were included in the review (Fig. 1). Five authors were contacted to request additional data that was not reported in the trial report; one [22] provided the data, one replied that the additional data were no longer available [23] and 3 [24-26] did not respond after three attempts.

\section{Characteristics of included trials}

The 15 included trials randomised a total of 1252 participants. Sample sizes of the trials ranged from 22 [24] to 250 [23]. Surgeries included gastrectomy or abdominal surgery $(n=4)$, spinal surgery $(n=2)$, orthopaedic lower limb surgery $(n=5)$, tracheostomy $(n=1)$, gynaecological surgery $n=1$, prostate resection $(n=1)$ and breast resection $(n=1)$. The trials investigated the effects of $\mathrm{z}$-drugs with analgesic medicines vs the same analgesic medicines alone $(n=4)$, melatonin vs placebo $(n=2)$, benzodiazepines vs placebo $(n=3)$ and benzodiazepines with analgesic medicines vs the same analgesic medicines alone $(n=6)$. In 10 trials, the intervention was administered orally, IV in 3 trials, IM in one and via an intranasal spray in one trial.

Pain intensity was reported in 13 trials. Opioid consumption was measured in 7 trials. Two [22, 27] declared financial support from the pharmaceutical industry. Descriptive characteristics of each trial are provided in Table 1. Results for secondary outcomes are described in Table 2 and reported in greater detail in Additional file 2.

\section{Study quality}

A visual presentation of the risk of bias for each trial included is presented in Table 3. The most common risks of bias were did not provide data on group comparability at baseline $(80 \%)$ and did not specify the method of allocation concealment $(80 \%)$.

\section{Other potential sources of bias Publication bias}

We did not assess publication bias with funnel plots because too few studies were included in the metaanalysis.

\section{Synthesis of results}

Data from 8 trials $[22-25,27-30]$ were combined in a meta-analysis.

The results from 7 trials $[26,31-36]$ were synthesised narratively due to heterogeneity of the type of intervention (i.e. different combinations of hypnotic medicines and other pain medicines), route of administration (i.e. oral, 


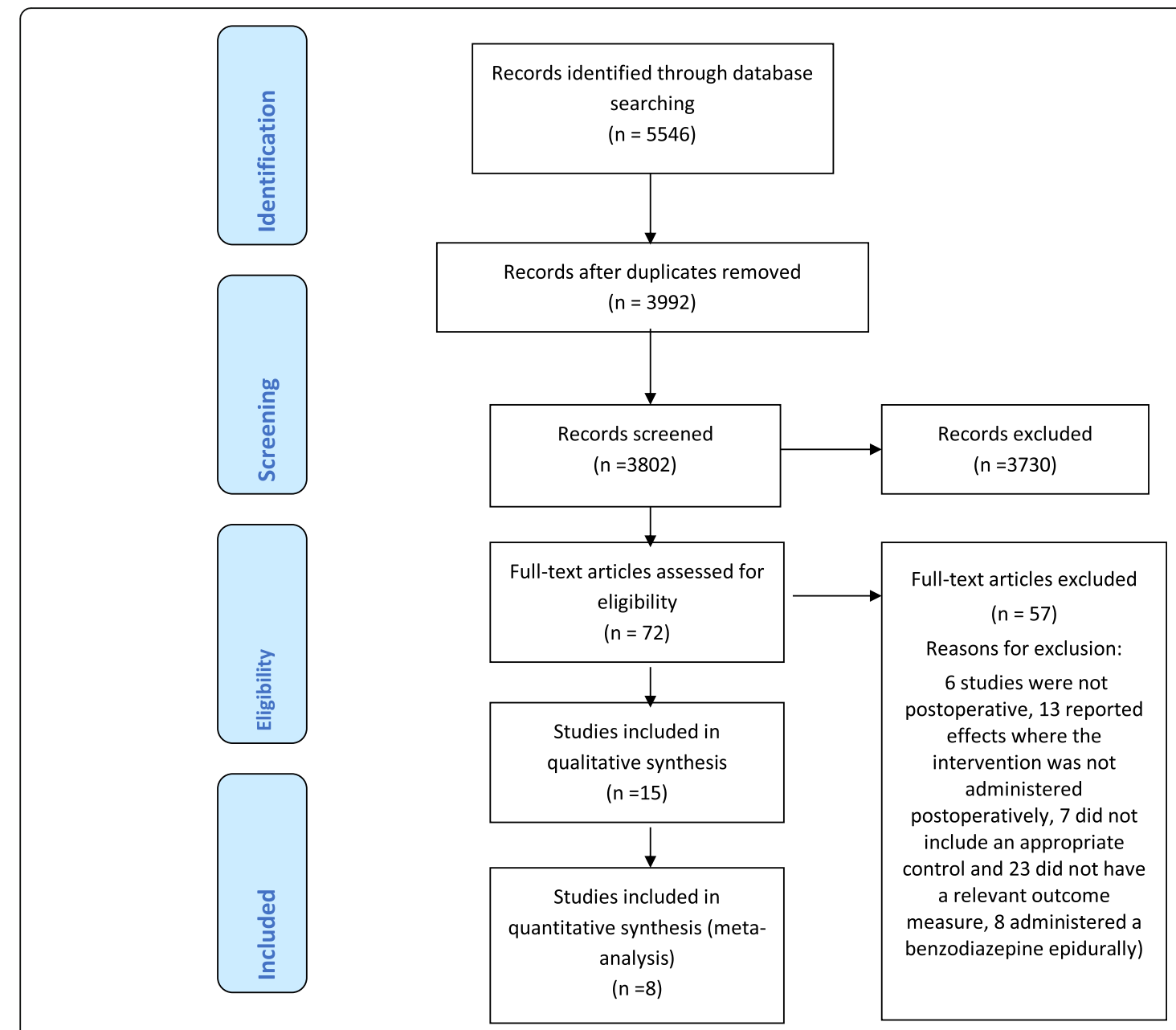

Fig. 1 PRISMA flow chart

IV, IM), comparison group (i.e. the same analgesic medicines or different analgesic medicines or placebo) or timing of outcomes assessment (immediately, short-term, medium-term, long-term).

\section{The main effect of hypnotic medicines on pain intensity The effect of $z$-drugs with analgesic medicines versus the same analgesic medicines alone (Fig. 2)}

There is moderate-quality evidence (downgraded due to imprecision) that in the immediate postoperative period ( 2 trials, $n=161[24,28]$ ) the effect of oral zolpidem 5/ $10 \mathrm{mg}$ (taken at night) with other analgesic medicines compared to the same analgesic medicines alone on pain intensity was not significant [WMD $-0.25, \mathrm{CI}-0.81$ to $0.31, p=0.38$.

There is moderate-quality evidence (downgraded due to imprecision) that in the short-term postoperative period ( 3 trials, $n=238[22,25,28]$ ) the effect of oral zolpidem (taken at night for seven nights) with other analgesic medicines significantly decreased pain intensity compared to the same analgesic medicines alone [WMD -1.06 , CI -1.48 to $-0.64, p \leq .01]$.

There is low-quality evidence (downgraded due to imprecision and inconsistency) that in the medium-term postoperative period ( 1 trial, $n=141$ [28]) the effect of oral zolpidem $5 \mathrm{mg}$ (taken at night for 14 nights) with other analgesic medicines significantly decreased pain intensity compared to the same analgesic medicines alone [WMD $-0.90, \mathrm{CI}-1.43$ to $-0.37, p \leq .01$ ].

\section{Melatonin versus placebo}

There is low-quality evidence (downgraded due to imprecision and inconsistency) that in the short-term postoperative period ( 1 trial, $n=121$ [33]) the effect of oral melatonin $5 \mathrm{mg}$ (taken at night for three nights) on pain intensity compared to placebo was not significant [WMD 0.10, CI -0.61 to $0.81, p=0.78$ ].

\section{Benzodiazepines versus placebo (Fig. 3)}

There is moderate-quality evidence (downgraded due to imprecision) that in the immediate post-operative period 


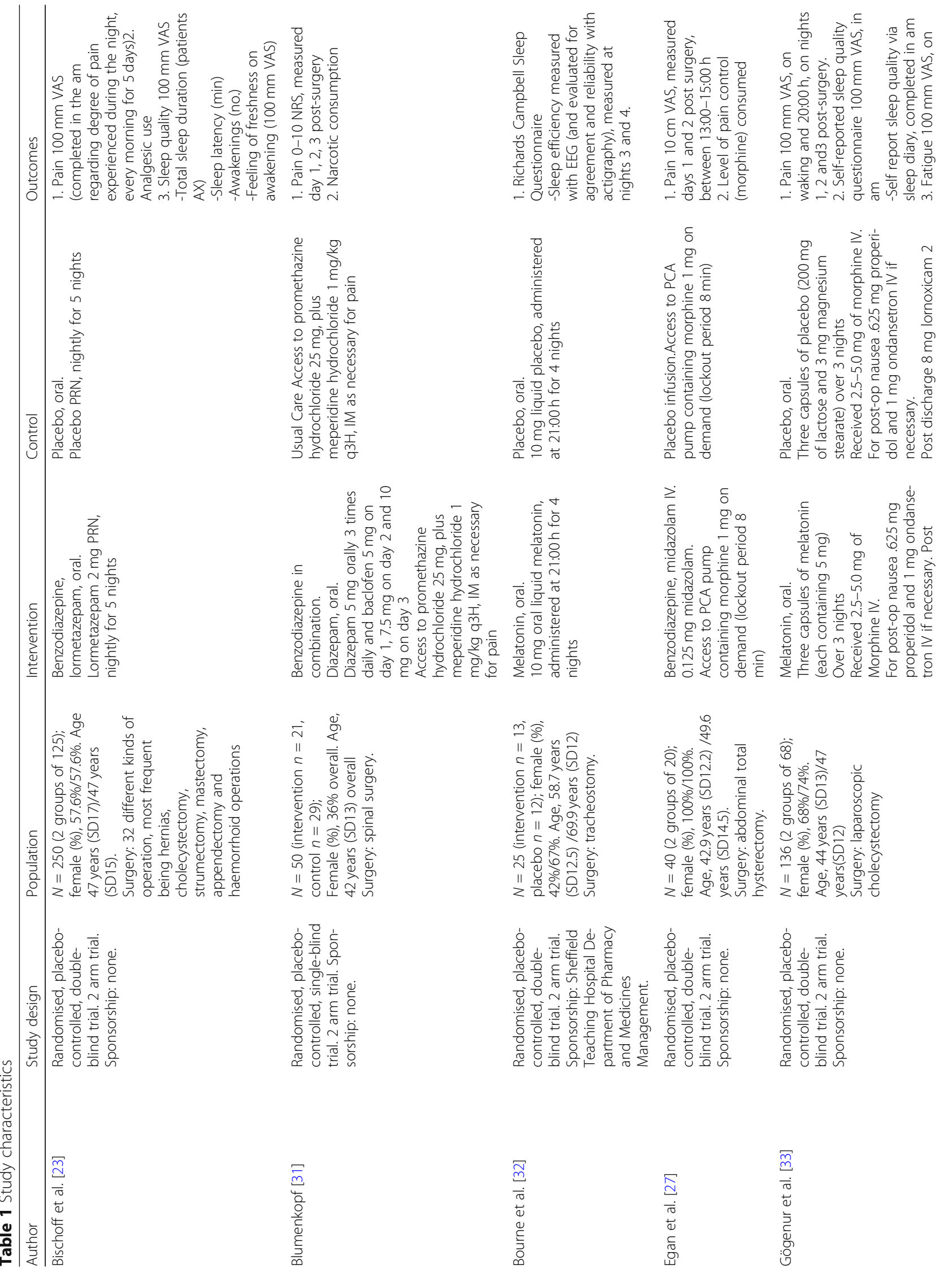




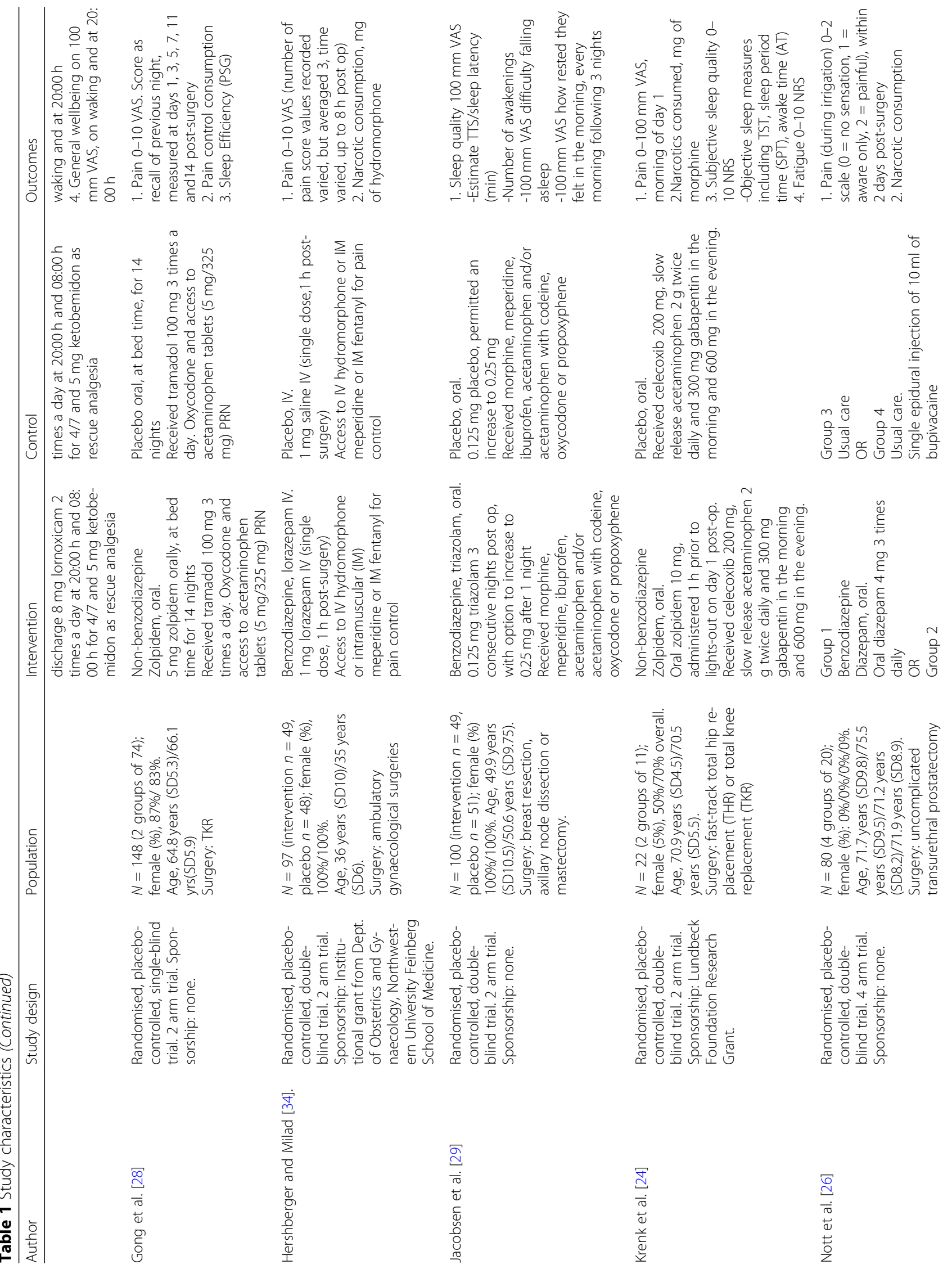




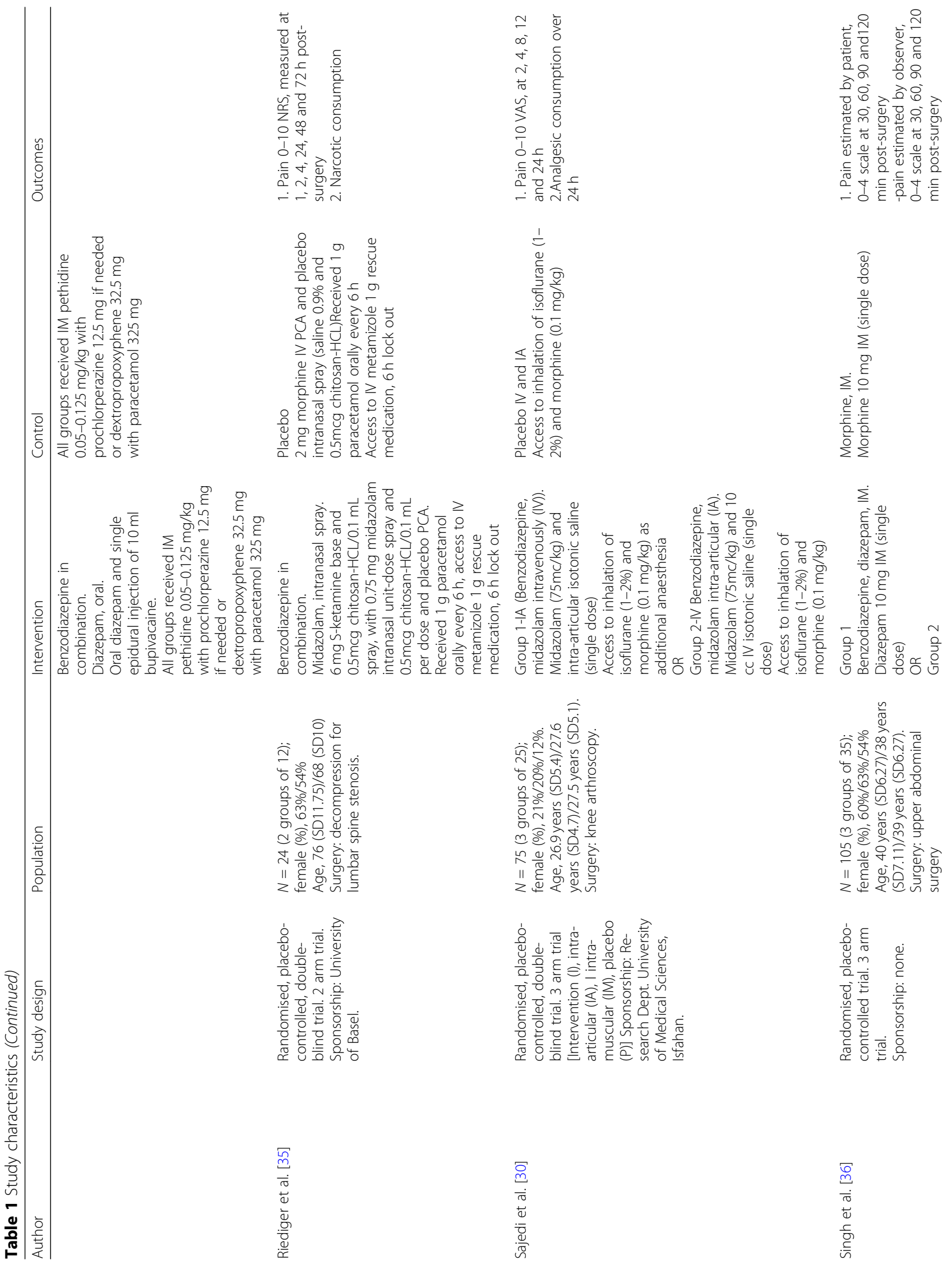



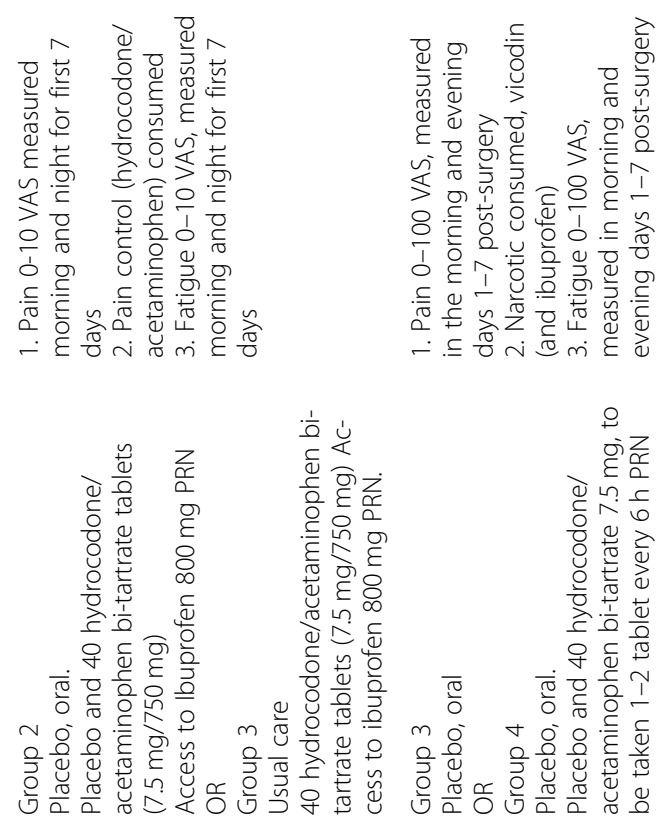

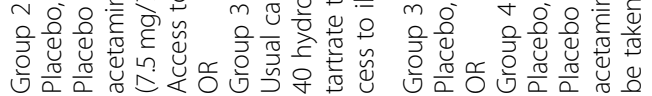
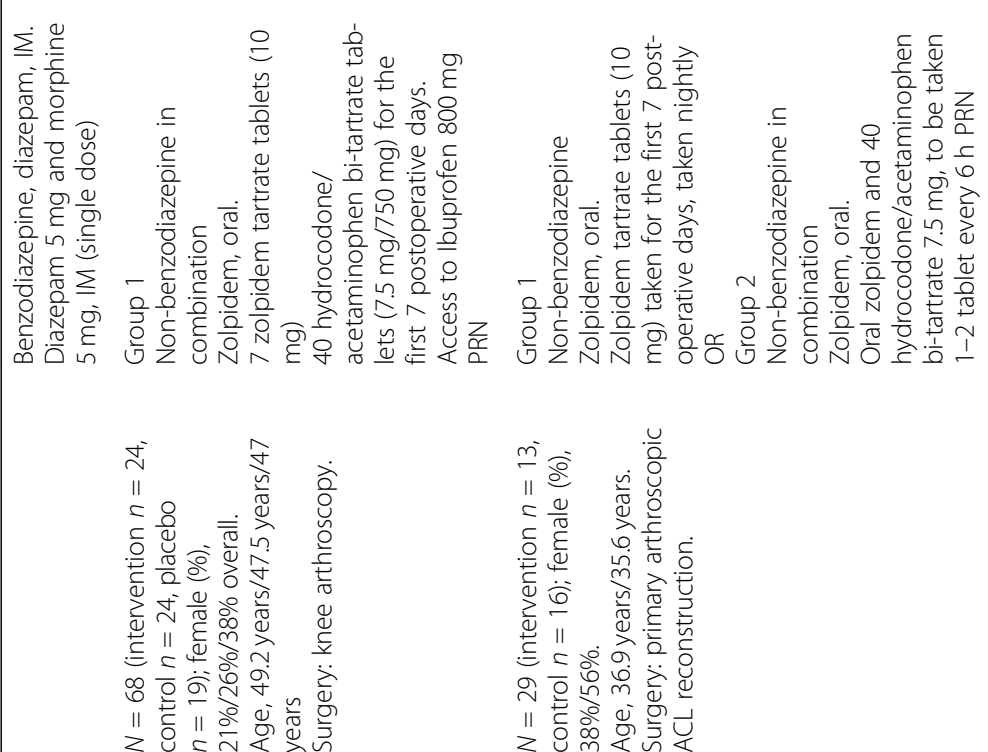

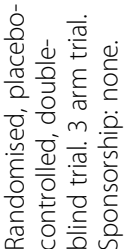

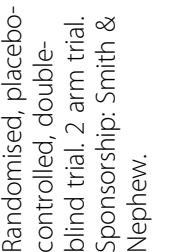


Table 2 Secondary outcomes

\begin{tabular}{|c|c|c|c|c|c|c|}
\hline & Time point & $n$ & Outcome & $\begin{array}{l}\text { Weighted mean } \\
\text { difference (WMD) }\end{array}$ & $\begin{array}{l}\text { Confidence } \\
\text { interval }\end{array}$ & $p$ value \\
\hline \multicolumn{7}{|l|}{ Sleep outcomes } \\
\hline \multirow{2}{*}{$\begin{array}{l}\text { Z-drugs in with analgesic medicines versus } \\
\text { the same analgesic medicines alone }\end{array}$} & Immediate & $20(24)$ & Sleep quality (0-10 NRS) & -1.60 & -2.91 to -0.3 & $p \leq .01$ \\
\hline & Immediate & $141(28)$ & Sleep efficiency (PSG) & -0.86 & 0.51 to 1.20 & $p \leq .01$ \\
\hline \multirow[t]{2}{*}{ Melatonin versus placebo } & Short-term & $24(35)$ & $\begin{array}{l}\text { Sleep quality (Richards } \\
\text { Campbell Questionnaire) }\end{array}$ & -0.09 & -0.28 to 0.09 & $p=0.32$ \\
\hline & Short-term & $121(31)$ & Sleep quality (0-100 VAS) & -0.09 & -0.27 to 0.45 & $p=0.51$ \\
\hline \multirow[t]{2}{*}{ Benzodiazepines versus placebo } & Immediate & $344(23)(30)$ & Sleep quality (0-100 VAS) & 1.14 & 1.63 to 0.65 & $p<.01$ \\
\hline & Short-term & $250(23)$ & Sleep quality (0-100 VAS) & 0.60 & 0 to 1.20 & $p=0.5$ \\
\hline \multicolumn{7}{|l|}{ Fatigue } \\
\hline \multirow{2}{*}{$\begin{array}{l}\text { Z-drugs with analgesic medicines versus } \\
\text { the same analgesic medicines alone }\end{array}$} & Immediate & $49(22)(24)$ & Fatigue (0-10 NRS) & -0.59 & -0.59 to 0.89 & $p=0.56$ \\
\hline & Short-term & $70(22)(25)$ & Fatigue (0-10 NRS) & 0.29 & -0.21 to 0.78 & $p=0.25$ \\
\hline Melatonin versus placebo & Immediate & $121(31)$ & Fatigue (0-100 VAS) & -0.40 & -1.16 to 0.36 & $p=0.30$ \\
\hline \multicolumn{7}{|l|}{ Wellbeing } \\
\hline & Immediate & $121(31)$ & General wellbeing (0-100 VAS) & -0.10 & -0.91 to 0.71 & $p=0.81$ \\
\hline
\end{tabular}

(2 trials, $n=89[27,30]$ ) the effect of an infusion of midazolam $(0.125 \mathrm{mg} / \mathrm{kg}$ or $0.075 / \mathrm{kg})$ on pain intensity compared to placebo was not significant [WMD - 0.00, CI -0.29 to $0.29, p=1.00]$.

There is low-quality evidence (downgraded due to imprecision and inconsistency) that in the immediate postoperative period ( 1 trial, $n=97$ [34]) the effect of intravenous lorazepam $1 \mathrm{mg}$ (4 times a day) was significantly less effective on pain intensity compared to placebo [WMD $1.00, \mathrm{CI} 0.22$ to $1.78, p \leq .01$ ]. This trial was not included in the meta-analysis due to the use of a different benzodiazepine.

\section{Benzodiazepines versus other analgesic medicines}

There is very low-quality evidence (downgraded due to high risk of bias, imprecision and inconsistency) that in the immediate postoperative period ( 1 trial, $n=105$ [36]) the effect of intramuscular diazepam $10 \mathrm{mg}$ (4 times a day) on pain intensity compared to intramuscular morphine $10 \mathrm{mg}$ (4 times a day) was not significant [WMD 2.00, CI 1.53 to 2.47, $\mathrm{p} \leq .01$ ].

\section{Benzodiazepines with other analgesic medicines versus the same analgesic medicines alone (not meta-analysed due to variability in the timing of outcomes and the control groups)}

There is very low-quality evidence (downgraded due to high risk of bias, imprecision and inconsistency) that in the immediate postoperative period ( 1 trial, $n=80$ [26]) the effect of oral diazepam, $4 \mathrm{mg}$ (3 times a day) with intramuscular pethidine and prochlorperazine, or oral dextropropoxyphene and paracetamol was significantly more effective on pain intensity compared to the same analgesic medicines alone [WMD - 4.0, CI - 5.55 to $-2.45, p \leq .01]$.

There is very low-quality evidence (downgraded due to high risk of bias, imprecision and inconsistency) that in the short-term postoperative period ( 1 trial, $n=50$ [31]) the effect of oral diazepam $5 \mathrm{mg}$ ( 3 times a day, for 3 days) with oral promethazine, pethidine and baclofen on pain intensity compared with promethazine and pethidine was not significant [WMD $-0.14, \mathrm{CI}-0.84$ to $1.21, p=0.21$ ]

There is low-quality evidence (downgraded due to imprecision and inconsistency) that in both the immediate and short-term postoperative period ( 1 trial, $n=22$ [35]) the effect of intranasal midazolam $0.75 \mathrm{mg}$ with $6 \mathrm{mg} \mathrm{S}$ Ketamine base and $0.5 \mathrm{mcg}$ chitosan-HCL/0.1 mL spray, and placebo patient-controlled analgesia (PCA) on pain intensity compared with $2 \mathrm{mg}$ morphine IV PCA and placebo intranasal spray (saline $0.9 \%$ and $0.5 \mathrm{mcg}$ chitosan-HCL) was not significant [immediate WMD $-0.70, \mathrm{CI}-1.59$ to $0.19, p=0.12$ and short-term WMD $-0.65, \mathrm{CI}-1.35$ to $0.05, p=0.07]$

\section{Effects of hypnotic medicines on opioid consumption Z-drugs with analgesic medicines versus the same analgesic medicines alone (not meta-analysed due to variability in the timing of outcomes and the control groups)}

There is low-quality evidence (downgraded due to imprecision and inconsistency) that in the immediate postoperative period ( 1 trial, $n=20$ [24]) oral zolpidem 5/10 mg (taken at night) with other analgesic medicines was not associated with opioid consumption (measured as the number of milligrammes of morphine consumed) 
O'Hagan et al. Systematic Reviews $\quad$ (2020) 9:206

Page 11 of 18

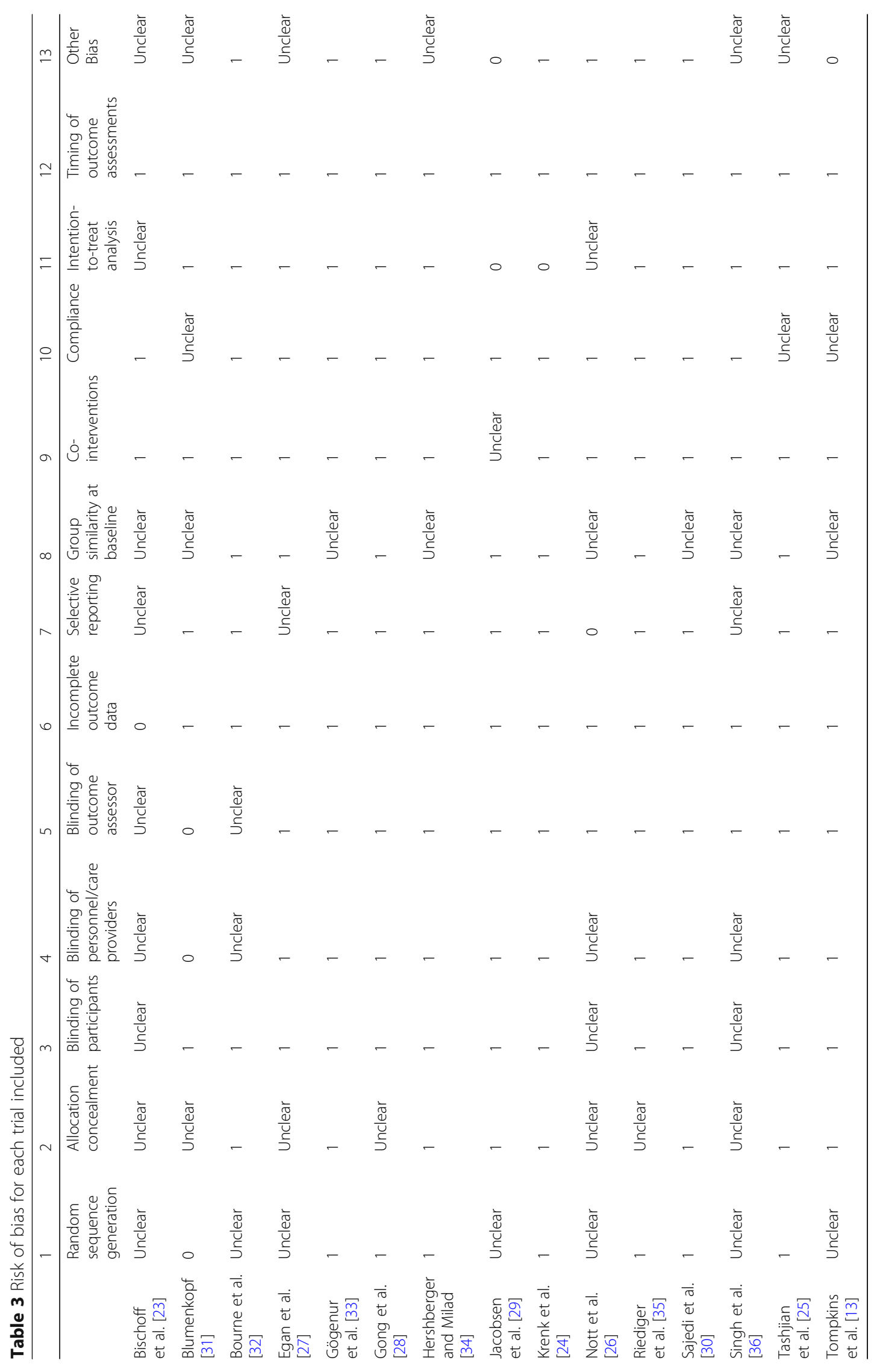




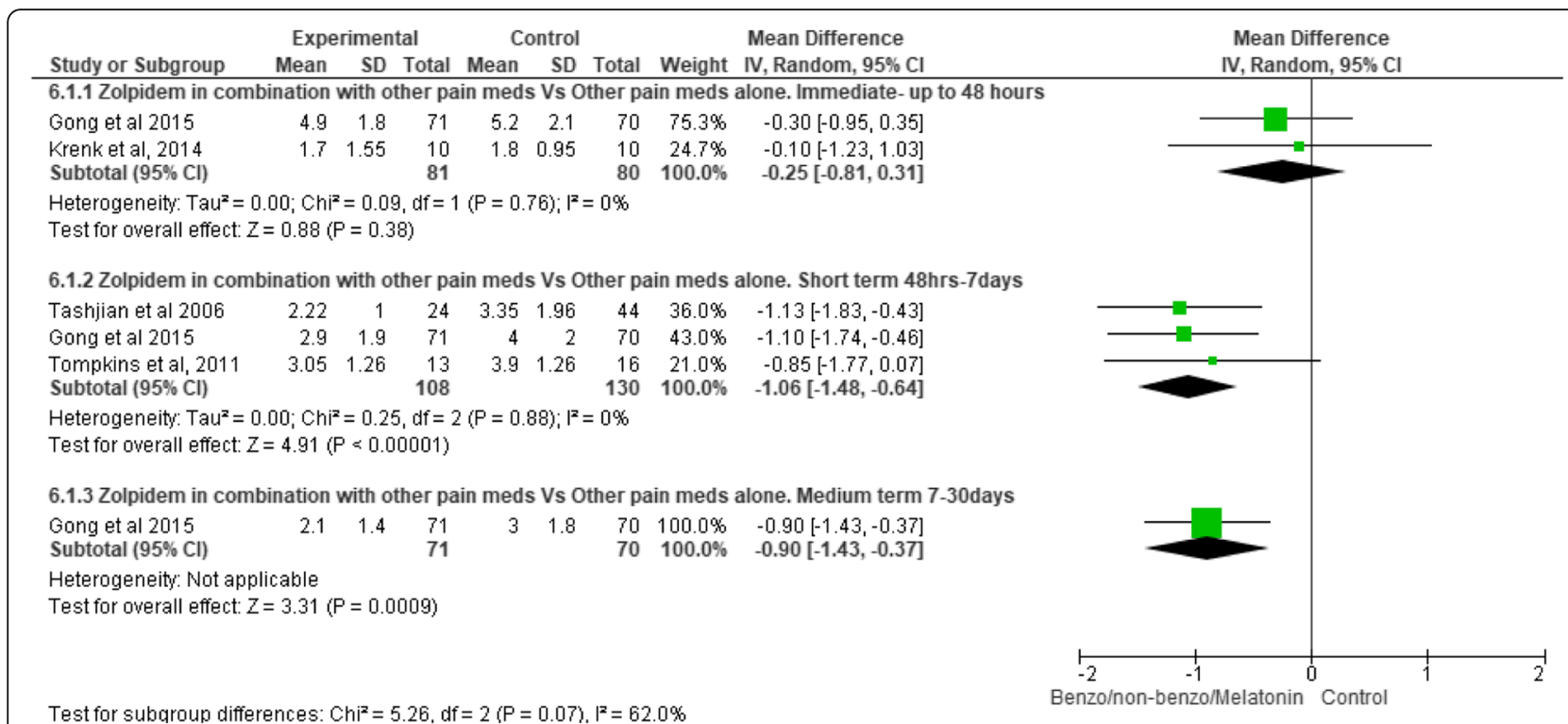

Fig. 2 Z-drugs with analgesic medicine versus the same analgesic medicines alone

compared to the same analgesic medicines alone [WMD -1.00 , CI -22.98 to $20.98, p=-0.93$ ].

There is moderate-quality evidence (downgraded due to imprecision) that in the short-term postoperative period ( 2 trials, $n=97[22,25]$ ), the effect of oral zolpidem (taken at night for seven nights) with other analgesic medicines significantly decreased opioid consumption (measured hydrocodone/acetaminophen bitartrate consumed) compared to the same analgesics medicines alone [WMD - 3.04, CI -5.73 to $-0.35, p=0.03$ ].

There is low-quality evidence (downgraded due to imprecision and inconsistency) that in the medium-term postoperative period ( 1 trial, $n=141$ [28]) the effect of oral zolpidem $5 \mathrm{mg}$ (at night for 14 nights) with other analgesic medicines significantly decreased opioid consumption (measured as the number of milligrammes of morphine consumed) compared to the same analgesic medicines [WMD - 116.90, CI - 131.88 to $-101.92, p<.01$ ]

\section{Benzodiazepines versus placebo}

There is moderate-quality evidence (downgraded due to imprecision) that in the immediate postoperative period
(2 trials, $n=89[27,30]$ ) the effect of an infusion of midazolam $(0.125 \mathrm{mg} / \mathrm{kg}$ or $0.075 / \mathrm{kg})$ was not associated with opioid consumption (measured as the number of milligrammes of morphine consumed) compared to placebo [WMD - 20.71, CI - 49.63 to 8.22, $p=0.16$ ].

The following trial was not added to the meta-analysis as it reported a different combination of hypnotic medicines.

There is very low-quality evidence (downgraded due to high risk of bias, imprecision and inconsistency) that in the immediate postoperative period ( 1 trial, $n=97$ [36]) the effect of intravenous lorazepam $1 \mathrm{mg}$ (4 times a day) was not associated with opioid consumption (measured as the number of milligrammes of fentanyl consumed) compared to placebo [WMD 0.20, CI - 0.10 to $0.50, p=0.20]$.

\section{Sub-group analyses \\ Effect of hypnotic medicines on pain intensity over time (hypnotic medicine by time interaction)}

The effect of each hypnotic medicine on pain intensity over time is presented in Table 4 and Fig. 4.

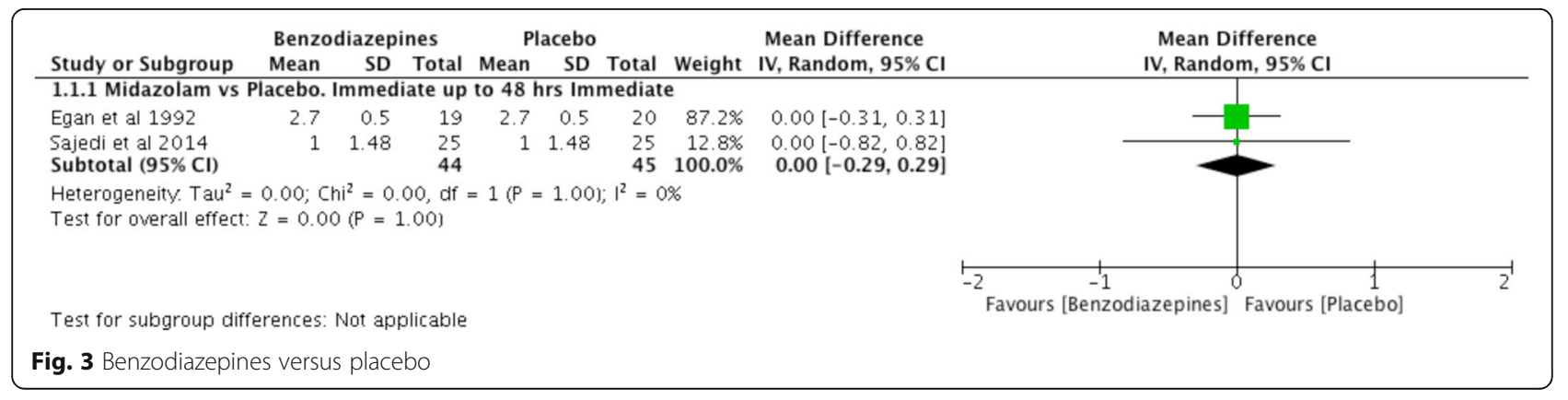


Table 4 Effectiveness of hypnotic medicines over time

\begin{tabular}{|c|c|c|c|c|}
\hline & $<12 \mathrm{~h}$ & $12-24 h$ & $24-36 h$ & $>48 \mathrm{~h}$ \\
\hline Zolpidem & $\begin{array}{l}\text { WMD }-0.1 \\
\mathrm{Cl}-0.39 \text { to } 0.19 \\
p \text { value }<0.01\end{array}$ & $\begin{array}{l}\text { WMD }-0.3 \\
C I-0.38 \text { to }-0.22 \\
p \text { value }<0.01\end{array}$ & $\begin{array}{l}\text { WMD }-1.49 \\
C I-1.63 \text { to }-1.34 \\
p \text { value }<0.01\end{array}$ & $\begin{array}{l}\text { WMD }-1.09 \\
C l-1.12 \text { to }-1.06 \\
p \text { value }<0.01\end{array}$ \\
\hline Melatonin & $\begin{array}{l}\text { WMD } 1.09 \\
\text { Cl } 0.77 \text { to } 1.40 \\
p \text { value }<0.01\end{array}$ & $\begin{array}{l}\text { WMD } 0.50 \\
\text { Cl } 0.18 \text { to } 0.82 \\
p \text { value }<0.01\end{array}$ & $\mathrm{n} / \mathrm{a}$ & $\mathrm{n} / \mathrm{a}$ \\
\hline Midazolam & $\begin{array}{l}\text { WMD }-0.32 \\
C l-0.38 \text { to }-0.27 \\
p \text { value }<0.01\end{array}$ & $\begin{array}{l}\text { WMD } 0.20 \\
\mathrm{Cl}-0.14 \text { to } 0.54 \\
p \text { value }<0.01\end{array}$ & $\begin{array}{l}\text { WMD } 1.39 \\
\text { Cl } 1.05 \text { to } 1.72 \\
p \text { value }<0.01\end{array}$ & $\mathrm{n} / \mathrm{a}$ \\
\hline Lorazepam & $\begin{array}{l}\text { WMD } 1.00 \\
\mathrm{Cl} 0.88 \text { to } 1.12 \\
p \text { value }<0.01\end{array}$ & $\mathrm{n} / \mathrm{a}$ & $\mathrm{n} / \mathrm{a}$ & $\mathrm{n} / \mathrm{a}$ \\
\hline
\end{tabular}

WMD weighted mean difference, $C l$ confidence interval, $n / a$ no measure for this time point

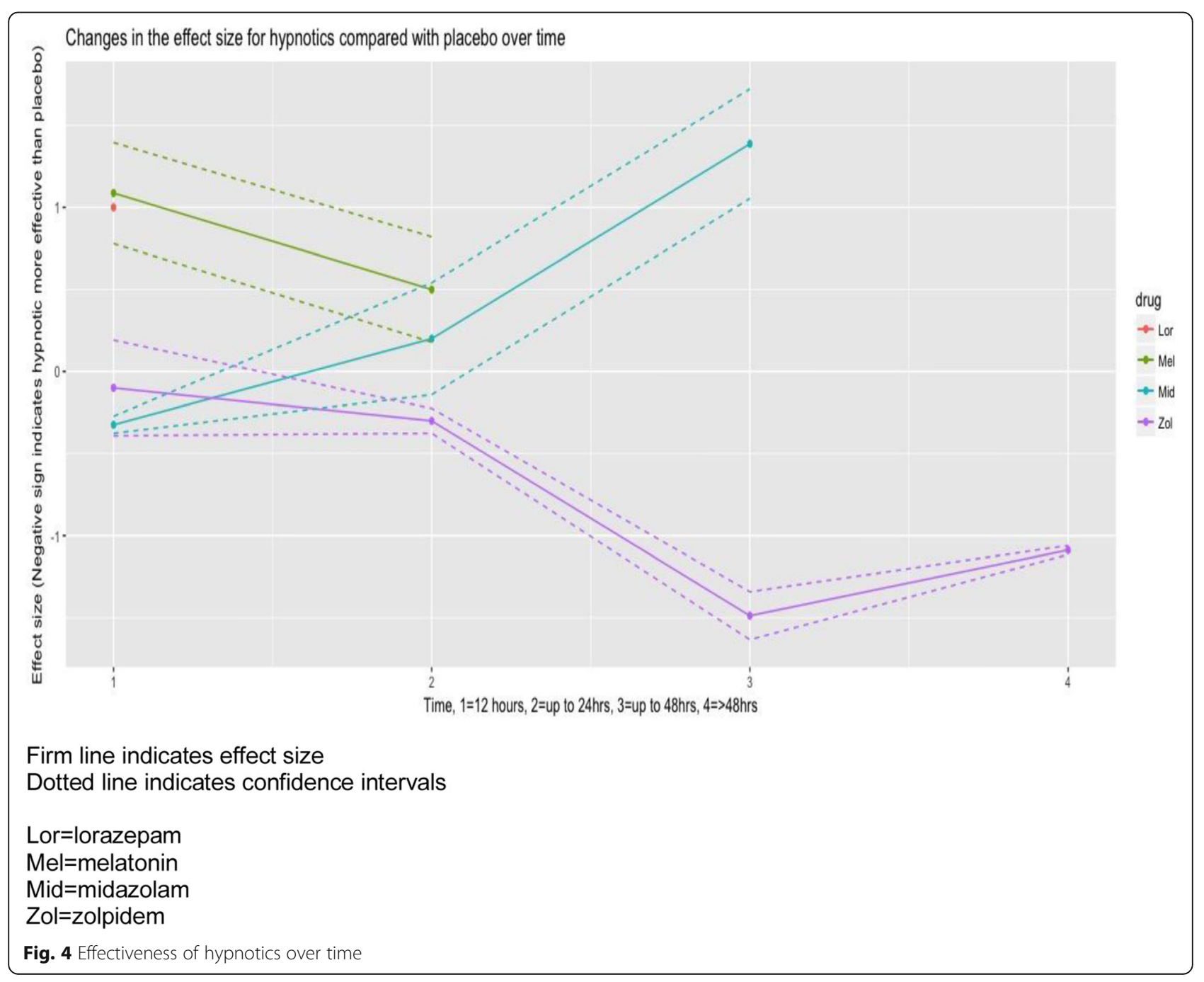


The effect of zolpidem on pain intensity achieves a clinically important difference of 1-point on a 10-point scale [37] from $24 \mathrm{~h}$ postoperatively. This clinically important difference in effect size of zolpidem compared to placebo is maintained at 7 days [WMD - 1.09, CI - 1.12 to $-1.06, p<0.01]$.

Melatonin is significantly less effective at reducing pain intensity than placebo at both $12 \mathrm{~h}$ [WMD 1.09, CI 0.77 to $1.40, p<0.01$ ] and $24 \mathrm{~h}$ [WMD 0.5 , CI 0.18 to $0.82, p<0.01]$.

Midazolam was more effective than placebo at reducing pain intensity up to $12 \mathrm{~h}$ postoperatively [WMD 0.32 , CI -0.38 to $0.27, p<0.01$. Midazolam is less effective than placebo at both $24 \mathrm{~h}$ [WMD 0.20 , CI 0.18 to $0.82, p<0.01$ ] and at $36 \mathrm{~h}$ post-operatively [WMD1.39, CI 1.05 to $1.72, p<0.01]$. Lorazepam was less effective than placebo at reducing pain intensity over time [WMD 1.00 , CI 0.88 to $1.12, p<0.01$ ].

\section{The effect of type of comparison medicine on the relationship between hypnotic medicines and pain intensity} The effect of hypnotic medicines on pain intensity varied depending on the type of comparison medicine. Overall, the effect of hypnotic medicines compared to placebo on pain intensity was not significant [WMD $=-0.29$, CI 0.98 to $0.39, p=0.19$ ]. Hypnotic medicines were not more effective at decreasing pain intensity compared to morphine [WMD $=0.68, \mathrm{CI}-0.70$ to $2.05, p=0.33$ ] Hypnotic medicines with analgesic medicines were significantly more effective at decreasing pain intensity than the same analgesics alone [WMD $=-2.14, \mathrm{CI}-3.52$ to $-0.76, p<0.01]$.

\section{The effect of route of administration on the relationship between hypnotic medicine and pain intensity}

Hypnotic medicines were significantly more effective at decreasing pain intensity when delivered orally [WMD = - 0.85, CI -1.58 to $-0.12, p=0.02$ ]. Hypnotics were not more effective at decreasing pain intensity when delivered via an infusion [WMD $=0.23, \mathrm{CI}-0.34$ to 0.81 , $p=0.43]$.

The trials in which the hypnotic medicines were delivered intranasally or intramuscularly were not compared to a placebo so were not included in the metaregression.

There were insufficient data to perform subgroup analysis to determine whether the effects on pain intensity were moderated by the duration of symptoms or type of surgery.

\section{Sensitivity analysis}

We were unable to conduct a sensitivity analysis to determine whether excluding trials of lower methodological quality or higher risk of bias affected the effects of the group comparisons due to the small number of trials. With one exception [23], the trials included in the meta-analysis had more endorsed quality items than those not included (Table 2).

A post hoc sensitivity analysis was conducted to assess the effect of increasing the "immediate period" to $72 \mathrm{~h}$ postoperatively. Only one trial had additional data for this time period [28]. The inclusion of these data did not change the results. There was no effect of nonbenzodiazepines with other analgesics on pain intensity up to $72 \mathrm{~h}$ postoperatively [WMD -0.71 , CI -1.67 to $0.24, p=0.14]$.

\section{Discussion}

\section{Summary of evidence}

This systematic review found moderate-quality evidence that the hypnotic medicine zolpidem, a z-drug, administered in the postoperative period, has an analgesic effect in the short- and medium-term. The effect size is greatest at $36 \mathrm{~h}$ postoperatively, and a clinically important difference of 1-point on a 10-point scale [37] is maintained 1 week postoperatively. This effect is apparent when the hypnotic medicine is co-administered with analgesic medicines and not when administered as monotherapy. This finding raises the possibility that $\mathrm{z}$-drugs could play an important adjuvant role for postoperative pain management.

Melatonin was not effective at reducing postoperative pain compared to placebo. The results for the effect of benzodiazepines with analgesic medicines on pain intensity are mixed. While one [26] trial reported a significant decrease in pain intensity immediately postoperatively, a second showed no effect [35].

Although we were unable to determine whether the effects of hypnotic medicines on pain intensity are moderated by the duration of symptoms or type of surgery due to a limited number of studies, we found that the effect of hypnotic medicines was increased when delivered orally. Hypnotic medicines have an adjunctive role; our results show they are effective at decreasing pain intensity when combined with other analgesic medicines. When participants who only received hypnotics were compared with participants only receiving placebo, there was not a significant change in pain intensity. Similarly, when participants only received hypnotics were compared with participants receiving morphine, there was not a significant change in pain intensity.

The strengths of this study are that, to our knowledge, this is the first systematic review to investigate the effect of hypnotic medicines on pain intensity, sleep quality and opioid consumption postoperatively. We supplemented the analysis by conducting a mixed-methods meta-regression to investigate whether the different 
medicine classes (z-drugs and benzodiazepines) had different effects on pain intensity over time (Fig. 4).

The findings can be used to guide future research. The current guidelines on postoperative pain management recommend "clinicians offer multimodal analgesia, or the use of a variety of analgesic medicines and techniques combined with non-pharmacological interventions, for the treatment of postoperative pain in children and adults" [7]. Emphasis is placed on the importance of minimising opioid therapy as the guideline recommends clinicians incorporate routine non-opioid analgesics. Alternatives to opioids for multimodal pain management are necessary to further decrease the opioid dependence and prevent chronic opioid use.

\section{Effect on postoperative pain intensity}

We found a weighted mean between-group difference of 1.5 on an 11-point scale for pain intensity when zolpidem with analgesics was compared to placebo zolpidem and the same analgesics at $36 \mathrm{~h}$ postoperatively. At 7 days postoperatively, this effect was 1.1. Although the absolute effects are modest, the reduction in pain intensity is in addition to that obtained from the other analgesics, suggesting a possible adjuvant role in postoperative pain management.

\section{Effect on opioid consumption}

The evidence for opioid-sparing effects of zolpidem was mixed. It is noteworthy that the larger trial, of higher quality [28], found that the intervention group, who were receiving zolpidem, required significantly less morphine over the course of the 14-day investigation period compared to the placebo group. These preliminary findings may have identified a promising alternative analgesic option with opioid-sparing effects.

\section{Effect on postoperative sleep outcomes}

Improved sleep quality was highlighted as an important outcome in a report on research gaps in clinical guidelines for the management of acute postoperative pain [38]. We investigated whether the use of a hypnotic drug administered postoperatively improved sleep outcomes. While we were unable to pool trials to determine the effect of z-drugs on sleep outcomes, our results suggest that benzodiazepines are more effective than placebo at improving sleep outcomes postoperatively.

This systematic review raises numerous questions for future research. Future research should consider other factors that influence pain intensity and sleep outcomes in the postoperative period, including anxiety, nursing interventions and environmental disturbances. Le Guen et al. [39], for instance, found that sleep quality was significantly improved postoperatively by the use of a simple intervention, earplugs and eye masks, which significantly decreased the total consumption of morphine.

Secondly, the benefits of melatonin may be underestimated in this trial due to the relatively small doses administered. Enhanced effects may be observed at higher dosages. Third, zolpidem's hypnotic effect is achieved by increasing GABA activity at the GABA-A receptor [40]. $\mathrm{Z}$ drugs unselectively bind to GABA-A subunits $1,2,3$ and 5 [41, 42]. GABA-A subunit 1 is known to be strongly associated with sedation [43]. There is promising evidence for the usefulness of hypnotic medications as part of an opioid-free multimodal balanced anaesthesia strategy to achieve sedation necessary for major surgery [44]. It is not known whether sedative hypnotics have an opioid sparing effect, though as both medicines are CNS depressant drugs, factors other than analgesia may be in play. These sedative effects of $z$-drugs should be investigated to maximise the parallels between achieving sedation and reducing pain intensity.

There are some limitations in our review that have reduced the strength of our conclusions. Firstly, sample sizes of the included trials were typically small; trials were conducted on samples of 22-250 participants; 9 trials included less than 100 participants. In our review, the risk of bias assessment suggested that over a quarter (4 out of 15) of the included trials were at high or unclear risk of bias. It is noteworthy that the most common potential sources of bias were that trials did not provide data on group comparability at baseline (53\%) and failed to specify the method of allocation concealment (47\%) or randomisation $(47 \%)$. Trials of zolpidem were typically of higher quality (Table 2).

No medicine is without hazards. Z-drugs are associated with an increased falls risk in older adults [36, 45]. The trials included in our systematic review did not find a significant difference in the rate or severity of adverse events between the intervention or control groups. Six of the included trials did not report on adverse events, and 9 reported non-significant adverse events. The most commonly reported adverse events included headache [32], nystagmus [35] and urinary retention [31]. Adverse events are known to be under-reported in clinical trials. Even if adverse events were adequately reported, we acknowledge that RCTs traditionally have limited capacity to evaluate the safety of a medicine [46]. As the trials included in this review have small sample sizes, short follow-ups and restrictive inclusion criteria, they are a poor method for assessing safety. Consequently, side effects may be under-reported [46]. This makes it difficult to conclusively weigh the benefits of these interventions against harms.

There are some concerns that hypnotics delay recovery and discharge postoperatively [47]. We were unable to evaluate this effect as none of the included trials reported time to discharge as an outcome. 
The trials we included that investigated the effect of zolpidem on pain intensity postoperatively were conducted on patients undergoing orthopaedic surgery. It is not clear whether the type of surgery or the patient population is important when investigating the effect of the hypnotic medications on pain intensity.

Finally, we conducted a meta-regression to investigate the efficacy of hypnotics in relation to how they were administered. The results are not clinically useful as zdrugs are routinely administered orally only.

\section{Clinical implications}

We found that zolpidem, in combination with other analgesics, significantly improved pain intensity and sleep quality postoperatively, with modest effects. We found a consistent decrease in pain intensity of more than 1 point on a 10-point scale when zolpidem with analgesic medicines was compared to a placebo between $24 \mathrm{~h}$ and 7 days postoperatively. Individually, the trials that investigated the effect of zolpidem on pain intensity postoperatively were of low-risk of bias, but when the evidence is taken as a whole, the strength of the evidence is reduced. This is due to the trials eligible for inclusion having small sample sizes. This is an exploratory study that adds to the body of literature as the first systematic review to investigate the effect of hypnotic medicines on pain intensity, sleep quality and opioid consumption postoperatively. It supports recent evidence that perioperative addition of melatonin or zolpidem may improve postoperative pain control [48]. Although the absolute effects are modest, the reduction in pain intensity, improvement in sleep quality and reduction in opioid consumption suggests that these medicines may have an adjuvant role in postoperative pain management.

\section{Conclusion}

This study has identified areas for future research, including the opioid-sparing effects of hypnotics and the potential benefit of addressing sleep quality to improve pain intensity postoperatively. The current results should be interpreted with caution due to lack of data on safety, a small number of trials included in the pooled effects and their sample sizes. In general, future studies should include a greater number of participants, a robust safety protocol and record time to discharge. Specifically, the effect of zolpidem on pain intensity postoperatively should be investigated as an adjunct to simple analgesic medicines only to understand the opioid-sparing potential of zolpidem.

\section{Supplementary information}

Supplementary information accompanies this paper at https://doi.org/10. 1186/s13643-020-01458-8.

Additional file 1. PRISMA Checklist
Additional file 2. Secondary Outcomes

Additional file 3. Search Terms

\section{Abbreviations}

CBT-I: Cognitive Behavioural Therapy-Insomnia; GABA: Gamma aminobutyric acid; IM: Intramuscular; IV: Intravenous; NRS: Numeric Rating Scale; NSAI

DS: Non-steroidal anti-inflammatory drugs; PCA: Patient-controlled analgesia; RCT: Randomised controlled trial; TKR: Total knee replacement; VAS: Visual analogue scale; WMD: Weighted mean difference; Z-drugs: A group of nonbenzodiazepine hypnotics

Acknowledgements

The authors would like to thank Matthew Fernandez, for his help with translating a paper from Spanish to English.

\section{Authors' contributions}

EO conceived the overview, provided methodological expertise and wrote the protocol. MH conceived the overview, provided methodological and statistical expertise. CM provided systematic review and methodological and clinical area expertise. CG provided systematic review methodological and clinical area expertise. SG provided systematic review methodological expertise. NB provided methodological expertise. JHM is the guarantor and conceived the overview, provided systematic review and clinical area expertise. All authors read, contributed to and approved the final version of the manuscript.

\section{Funding}

This study was not funded or sponsored.

\section{Availability of data and materials}

The datasets used and/or analysed during the current study are available from the corresponding author on reasonable request.

Ethics approval and consent to participate

We did not require ethics approval to conduct this systematic review

Consent for publication

Not applicable

Competing interests

The authors have no conflicts of interest to declare.

\section{Author details}

${ }^{1}$ Centre for Pain IMPACT, Neuroscience Research Australia, Randwick, NSW 2031, Australia. ${ }^{2}$ Prince of Wales Clinical School, The University of New South Wales, Randwick, NSW 2031, Australia. ${ }^{3}$ CIRUS, Centre for Sleep and Chronobiology, Woolcock Institute of Medical Research, The University of Sydney, Glebe, NSW 2037, Australia. ${ }^{4}$ Faculty of Medicine and Health, The University of Sydney, Camperdown, NSW 2050, Australia. ${ }^{5}$ Gustin Pain Imaging Laboratory, School of Psychology, The University of New South Wales, Randwick, NSW 2031, Australia. ${ }^{6}$ Stats Central, University of New South Wales, Randwick, NSW 2031, Australia. ${ }^{7}$ School of Medical Science, University of New South Wales, Randwick, NSW 2031, Australia.

Received: 1 June 2020 Accepted: 14 August 2020

Published online: 03 September 2020

\section{References}

1. Gan TJ, Habib AS, Miller TE, White W, Apfelbaum JL. Incidence, patient satisfaction, and perceptions of post-surgical pain: results from a US national survey. Curr Med Res Opin [Internet]. 2014;30(1):149-60. Available from: http://www.ncbi.nlm.nih.gov/pubmed/24237004

2. Devin CJ, McGirt MJ. Best evidence in multimodal pain management in spine surgery and means of assessing postoperative pain and functional outcomes. J Clin Neurosci [Internet]. 2015;22(6):930-8. Available from: http://linkinghub.elsevier.com/retrieve/pii/S0967586815000156.

3. Mitchell $\mathrm{G}$. Review article reducing the pain : a systematic review of postdischarge analgesia following elective orthopedic surgery. Pain Med. 2012;13:711-27. 
4. Kehlet $H$, Jensen TS, Woolf CJ. Persistent postsurgical pain: risk factors and prevention. Lancet. 2006;367(9522):1618-25.

5. Rawal N. Current issues in postoperative pain management. Eur J Anaesthesiol [Internet]. 2015;1. Available from: http://content.wkhealth.com/ linkback/openurl?sid=WKPTLP:landingpage\&an=00003643-900000000-98869.

6. Althaus A, Arránz Becker O, Neugebauer E. Distinguishing between pain intensity and pain resolution: using acute post-surgical pain trajectories to predict chronic post-surgical pain. Eur J Pain (United Kingdom). 2014;18(4) $513-21$

7. Chou R, Gordon DB, de Leon-Casasola OA, Rosenberg JM, Bickler S, Brennan $T$, et al. Management of postoperative pain: a clinical practice guideline from the American Pain Society, the American Society of Regional Anesthesia and Pain Medicine, and the American Society of Anesthesiologists' Committee on Regional Anesthesia, Executive Commi. J Pain [Internet]. 2016;17(2):131-57. Available from: http://linkinghub.elsevier com/retrieve/pii/S1526590015009955.

8. Sun EC, Darnall B, Baker LC, Mackey S. Incidence of and risk factors for chronic opioid use among opioid-naive patients in the postoperative period. JAMA Intern Med [Internet]. 2016;94305(9):1-8. Available from: http://archinte.jamanetwork.com/article.aspx?doi=10.1001/jamainternmed.2 016.3298\%5Cn, http://www.ncbi.nlm.nih.gov/pubmed/27400458.

9. Glare P, Aubrey KR, Myles PS. Postoperative pain management and opioids 1 Transition from acute to chronic pain after surgery [Internet]. Vol. 393, www.thelancet.com. 2019 [cited 2019 Apr 16]. Available from: www. thelancet.com.

10. Neuman MD, Bateman BT, Wunsch H, and B. Postoperative pain management and opioids 2 Inappropriate opioid prescription after surgery [Internet]. Vol. 393, Lancet. 2019 [cited 2019 Apr 16]. Available from: www.thelancet.com.

11. Colvin LA, Bull F, Hales TG. Series Postoperative pain management and opioids 3 Perioperative opioid analgesia-when is enough too much? A review of opioid-induced tolerance and hyperalgesia [Internet]. Vol. 393, www.thelancet.com. 2019 [cited 2019 Apr 16]. Available from: www. thelancet.com.

12. Chouchou F, Khoury S, Chauny J-M, Denis R, Lavigne G. Postoperative sleep disruptions: a potential catalyst of acute pain? Sleep Med Rev [Internet]. 2014;18(3):253-62 Available from: https://doi.org/10.1016/j. smrv.2013.07.002

13. Alsaadi SM, MCAuley JH, Hush JM, Lo S, Bartlett DJ, Grunstein RR, et al. The bidirectional relationship between pain intensity and sleep disturbance/ quality in patients with low back pain. Clin J Pain [Internet]. 2014/01/24. 2014;30(9):755-65. Available from: http://graphics.tx.ovid.com/ovftpdfs/ FPDDNCGCODPLEA00/fs046/ovft/ive/gv023/00002508/00002508-2014 09000-00004.pdf.

14. Liberati A, Altman DG, Tetzlaff J, Mulrow C, Gotzsche PC, loannidis JPA, et al. The PRISMA statement for reporting systematic reviews and meta-analyses of studies that evaluate health care interventions: explanation and elaboration. J Clin Epidemiol. 2009;62(10):e1-34.

15. Lyphout C, Yates C, Margolin ZR, Dargan PI, Dines AM, Heyerdahl F, et al. Presentations to the emergency department with non-medical use of benzodiazepines and Z-drugs: profiling and relation to sales data. Eur J Clin Pharmacol. 2019;75(1):77-85.

16. Hudcova J, McNicol E, Quah C, Lau J, Carr DB. Patient controlled opioid analgesia versus conventional opioid analgesia for postoperative pain. Cochrane Database Syst Rev. 2006:4(4):1-126.

17. Mason M, Chong K, Smith I. Effect of opioid, hypnotic and sedating medications on sleep-disordered breathing in adults with obstructive sleep apnoea. Cochrane Database Syst Rev [Internet]. 2014;7(7):1-65. Available from: http://ovidsp.ovid.com/ovidweb.cgi? T=JS\&CSC=Y\&NEWS=N\&PAGE = fulltext\&D $=$ coch\&AN $=00075320-100000000-09463 \mathrm{http}: / / \mathrm{sfx}$. scholarsportal.info/uhn?sid = OVID:cochdb\&id = pmid:\&id $=$ doi:\&issn $=$ \&isbn $=$ \&volume $=$ \&issue $=4 \&$ spage $=$ \&pages $=$ \&date $=2014 \&$ title $=$ Cochrane+Database+of+System.

18. Higgins JP, Altman DG, Gotzsche PC, Juni P, Moher, David, Oxman, Andrew, et al. The Cochrane Collaboration's tool for assessing risk of bias in randomised trials. BMJ [Internet]. 2011 [cited 2019 Apr 11]; Available from: http://www.bmj.com/content/343/bmj.d5928/suppl/DC1.

19. Collaboration TC. Review Manager (RevMan) Version 5.3. 2014.

20. Team RC. R: a language and environment for statistical computing (Version 3.4. 2)[Computer software]. In: $R$ foundation for statistical computing [Internet]. 2017. Available from: http://www.r-project.org/.
21. Mueller PS, et al. Annals of nternal Medicine Ethical issues in stopping randomized trials early because of apparent benefit. Ann Intern Med. 2007; 146:878-81.

22. Tompkins M, Plante M, Monchik K, Fleming B, Fadale P. The use of a nonbenzodiazepine hypnotic sleep-aid (Zolpidem) in patients undergoing ACL reconstruction: a randomized controlled clinical trial. Knee Surgery, Sport Traumatol Arthrosc. 2011;19(5):787-91.

23. Bischoff RC, Schratzer M, Anders A. The normalization of sleep after surgical procedures, with and without the use of a hypnotic benzodiazepine. Curr Ther Res. 1985;38(1):3-14.

24. Krenk L, Jennum P, Kehlet $H$. Postoperative sleep disturbances after zolpidem treatment in fast-track hip and knee replacement. J Clin Sleep Med. 2014;10(3):321-6.

25. Tashjian RZ, Banerjee R, Bradley MP, Alford W, Fadale PD. Zolpidem reduces postoperative pain, fatigue, and narcotic consumption following knee arthroscopy: a prospective randomized placebo-controlled double-blinded study. J Knee Surg. 2006;19(2):105-11.

26. Nott $M$, Jameson $P$, Julious $S$. Diazepam for relief of irrigation pain after transurethral resection of the prostate. Eur J Anaesthesiol. 1997;14(2): 197-200.

27. Egan K, Ready LB, Nessly M, Greer BE. Self-administration of midazolam for postoperative anxiety: a double blinded study. Pain. 1992;49(1):3-8.

28. Gong L, Wang Z. Sleep quality effects recovery after total knee arthroplasty (TKA)-a randomized, double-blind, controlled study. J Arthroplasty [Internet]. 2015;30(11):1897-901 Available from: http://www.sciencedirect. com/science/article/pii/S088354031500131X.

29. Jacobsen PB, Massie MJ, Kinne DW, Holland JC. Hypnotic efficacy and safety of triazolam administered during the postoperative period. Gen Hosp Psychiatry. 1994;16(6):419-25.

30. Sajedi P, Nemati M, Mosavi SH, Honarmand A, Safavi MR. A randomized controlled trial for the effectiveness of intraarticular versus intravenous midazolam on pain after knee arthroscopy. J Res Med Sci. 2013;19(May):439-444.

31. Blumenkopf B. Combination analgesic-antispasmodic therapy in postoperative pain. Spine (Phila Pa 1976). 1987;384-7.

32. Bourne RS, Mills GH, Minelli C. Melatonin therapy to improve nocturnal sleep in critically ill patients: encouraging results from a small randomised controlled trial. Crit Care. 2008;12(2):R52.

33. Gögenur I, Kücükakin B, Bisgaard T, Kristiansen V, Hjortsø NC, Skene DJ, et al. The effect of melatonin on sleep quality after laparoscopic cholecystectomy: a randomized, placebo-controlled trial. Anesth Analg. 2009;108(4):1152-6.

34. Hershberger JM, Milad MP. A randomized clinical trial of lorazepam for the reduction of postoperative urinary retention. Obstet Gynecol. 2003;102(2): 311-6.

35. Riediger C, Haschke M, Bitter C, Fabbro T, Schaeren S, Urwyler A, et al. The analgesic effect of combined treatment with intranasal S-ketamine and intranasal midazolam compared with morphine patient-controlled analgesia in spinal surgery patients: a pilot study. J Pain Res. 2015;8:87-94.

36. Singh PN, Sharma P, Gupta PK, Pandey K. Clinical evaluation of diazepam for relief of postoperative pain. Br J Anaesth. 1981;53(8):831-6.

37. Busse JW, Bartlett SJ, Dougados M, Johnston BC, Guyatt GH, Kirwan JR, et al. Optimal strategies for reporting pain in clinical trials and systematic reviews: recommendations from an OMERACT 12 workshop [Internet]. J Rheumatol. 2015;42. Available from: http://www.jrheum.org/content/42/10/1962, http:// www.jrheum.org/alerts1.SignupforTOCsandotheralerts, http://jrheum.com/ faqwww.jrheum.orgwww.jrheum.orgDownloadedfrom. [cited 2018 Aug 17].

38. Gordon DB, de Leon-Casasola OA, Wu CL, Sluka K, Brennan T, Chou R. Research gaps on practice guidelines for acute postoperative pain management in adults: findings from a review of the evidence for an American Pain Society Clinical Practice Guideline. J Pain [Internet]. 2015; 17(2016):158-66. Available from: http://linkinghub.elsevier.com/retrieve/pii/ S1526590015009967.

39. Le Guen M, Nicolas-Robin A, Lebard C, Arnulf I, Langeron O. Earplugs and eye masks vs routine care prevent sleep impairment in post-anaesthesia care unit: a randomized study. Br J Anaesth. 2014;112(1):89-95.

40. Gunja N. The clinical and forensic toxicology of Z-drugs. J Med Toxicol. 2013;9(2):155-62.

41. Döble A. The mechanism of action of zopiclone. Eur psychiarty. 1995;10:117-28.

42. Brun J. Zopiclone, a cyclopyrrolone hypnotic: review of properties. Pharmacol Biochem Behav. 1988;29(4):831-2.

43. Savic M, Huang $S$, Furtmuller R, Clayton T, Huck S, Obradovic D, et al. Are GABAA receptors containing a5 subunits contributing to the sedative 
properties of benzodiazepine site agonists? Neuropsychopharmacology. 2008;33(2):332-9.

44. Brown EN, Pavone K, Naranjo M. Multimodal general anesthesia: theory and practice. Anesth Analg. 2018;127(5):1246-58.

45. Diem SJ, Ewing SK, Stone KL, Ancoli-lsrael S, Redline S, Ensrud KE. Use of non-benzodiazepine sedative hypnotics and risk of falls in older men. J Gerontol Geriatr Res [Internet]. 2014 [cited 2018 Aug 20];3(3):158. Available from: https://www.ncbi.nlm.nih.gov/pmc/articles/PMC4289612/pdf/nihms-64 8697.pdf.

46. Brewer T, Colditz GA. Current perspectives and future needs. JAMA. 1999;9: 824-9.

47. Bilotta F, Doronzio A, Stazi E, Titi L, Zeppa IO, Cianchi A, et al. Early postoperative cognitive dysfunction and postoperative delirium after anaesthesia with various hypnotics: study protocol for a randomised controlled trial-The PINOCCHIO trial. Trials [Internet]. 2011 [cited 2019 Apr 4]; 12(170). Available from: http://www.trialsjournal.com/content/12/1/170.

48. Bjurström MF, Irwin MR. Perioperative pharmacological sleep-promotion and pain control: a systematic review. Pain Pract. 2019;19(5):552-69.

\section{Publisher's Note}

Springer Nature remains neutral with regard to jurisdictional claims in published maps and institutional affiliations.

Ready to submit your research? Choose BMC and benefit from:

- fast, convenient online submission

- thorough peer review by experienced researchers in your field

- rapid publication on acceptance

- support for research data, including large and complex data types

- gold Open Access which fosters wider collaboration and increased citations

- maximum visibility for your research: over $100 \mathrm{M}$ website views per year

At $\mathrm{BMC}$, research is always in progress.

Learn more biomedcentral.com/submissions 